\title{
121. AMBROSIA ARTEMISIIFOLIA L., NUEVA ESPECIE PARA LA FLORA ALÓCTONA INVASORA DE ANDALUCÍA (ESPAÑA)
}

\author{
María del Mar TRIGO y José GARCÍA-SÁNCHEZ
}

\begin{abstract}
Ambrosia artemisiifolia, a new species for the aloctonous invasive flora of Andalusia (Spain)
\end{abstract}
Palabras clave. Ambrosia artemisiifolia, planta invasora, Andalucía, España.

Key words. Ambrosia artemisiifolia, invasive plant, Andalusia flora, Spain.

MÁlAGA. Mijas. Carretera Fuengirola-Mijas. Urbanización La Casita Colorada. 30SUF5349. En un arriate junto con plantas cultivadas. 6-VI-2006. M. Mar Trigo. MGC 63794.

Ambrosia artemisiifolia es una planta anual, originaria de Norteamérica e introducida en Europa hacia 1865 (Sanz Elorza et al., 2004), en donde se instala en campos de cultivo arados, arriates, bordes de carreteras y otros lugares ligados a la actividad humana. $\mathrm{Su}$ dispersión se ve favorecida por las actividades agrícolas, la maquinaria y el compostaje, habiéndose encontrado sus semillas en el pienso para animales ya que apenas se diferencian de las de algunas gramíneas forrajeras (Bohren, 2006). Debido al elevado potencial alergógeno de sus granos de polen (Rybncek \& Jäger, 2001), A. artemisiifolia es considerada un serio problema de salud pública, no sólo en Norteamérica sino en los países en los que se encuentra naturalizada, especialmente en el este y centro de Europa donde en algunos países coloniza casi $90 \%$ del territorio (Bohren, 2006) y en donde se están llevando a cabo importantes campañas de erradicación.

Dana et al. (2003), consideran a esta especie con un claro comportamiento invasor, aunque de momento sólo se ha encontrado en ambientes altamente modificados. Florece de junio a noviembre y según Sanz Elorza et al.
(2004), se trata de una planta pionera, bien adaptada para colonizar terrenos donde la vegetación natural ha sido eliminada. Se reproduce exclusivamente por semillas de dispersión endozoócora (aves), hidrócora, anemócora y antropócora, que suelen germinar a partir del mes de mayo.

A. artemisiifolia ha sido citada en la Península Ibérica en las localidades de Santander (Laínz \& Loriente, 1983); Arija, Burgos, (Aedo et al., 2001) y O Grove y Raxó, en Pontevedra, (García Martínez, 1985) y los únicos pliegos que actualmente aparecen recogidos en los registros del GBIF (Global Biodiversity Information Facility) corresponden a las localidades de Santander (C. Aedo. 6.IX.1982. MA 599582-1) y Ortuella, Bilbao, (J.A. Campos. 15.X.1997. MA 625636-1). En el caso que nos ocupa sólo se encontró un ejemplar aislado (fig. 1), en plena floración, probablemente originado a partir de semillas introducidas con el compost con que suele mezclarse la tierra de jardín y creemos que se trata de la primera cita para Andalucía y para la Península Ibérica fuera de zona septentrional. Dado su comportamiento invasor y su elevado poder alergógeno, recomendamos estar alerta con el fin de detectar su presencia en otras localidades del sur de la Península.

AGRADECIMIENTOS. A E. D. Dana Sánchez por facilitarnos información acerca de la 


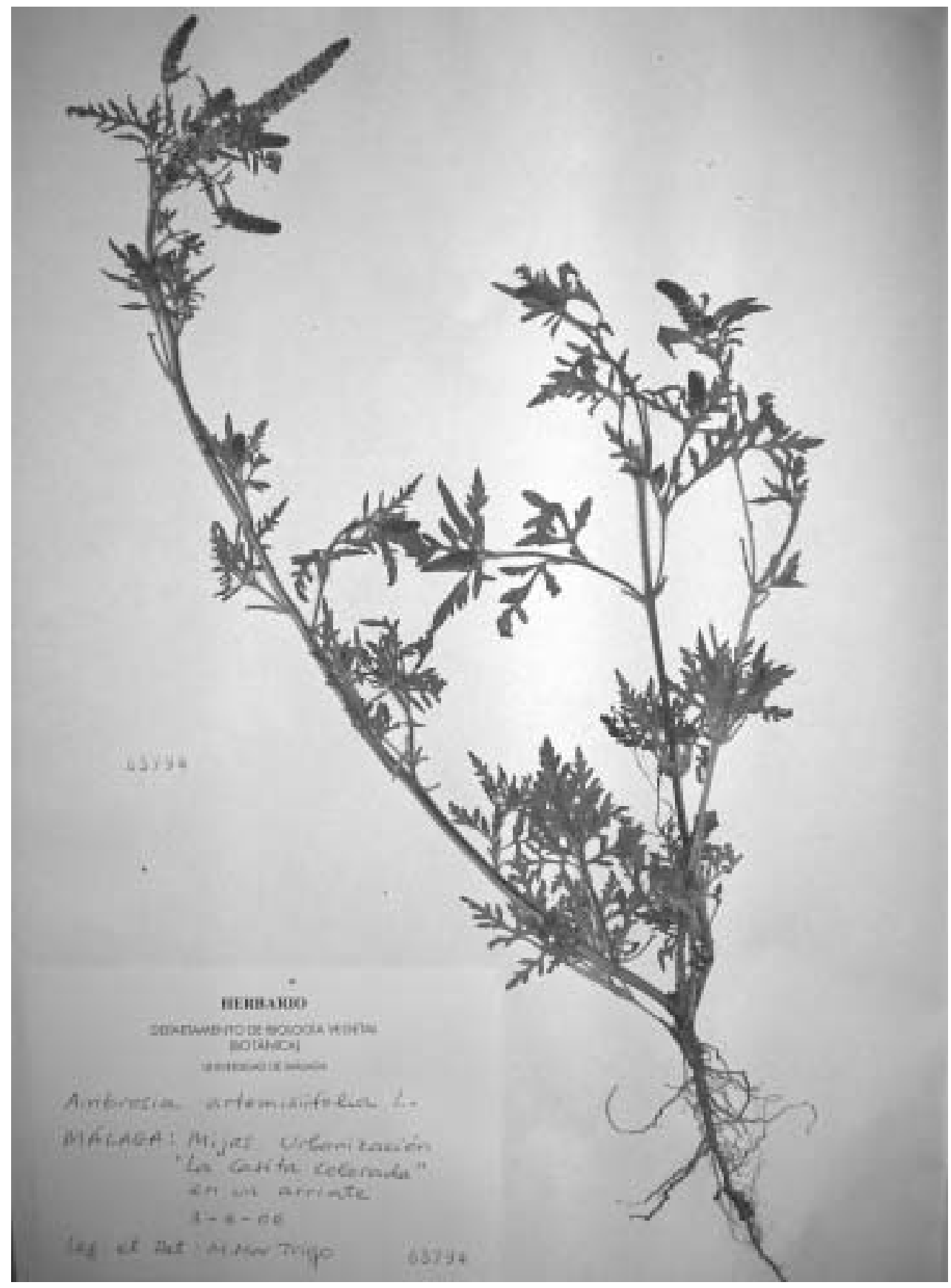

Figura 1. Ambrosia artemisiifolia L. (MGC 63794). 
distribución de esta especie y a G. Blanca que realizó la fotografía del pliego que, en el momento de redactar esta nota, se encontraba en préstamo en el Herbario de la Universidad de Granada.

\section{BIBLIOGRAFÍA}

AEDO, C., J. J. ALDASORO, J. M. ARGÜELLES, L. CARLÓN, A. DÍEZ RIOL, G. GÓMEZ CASARES, J. M. GONZÁLEZ DEL VALLE, A. GUILLÉN OTERINO, M. LAÍNZ, G. MORENO MORAL, J. PATALLO y O. SÁNCHEZ PEDRAJA -2001- Contribuciones al conocimiento de la flora cantábrica, V. Bol. Cien. Nat. R.I.D.E.A. 47: 7-52.

C. BOHREN, G. MERMILLOD \& N. DELABAYS -2006-. Common ragweed (Ambrosia artemisiifolia L.) in Switzerland: development of a nationwide concerted action. Journal of Plant Diseases and Protection, special issue XX: 497-503.

DANA, E.D., M. SANZ-ELORZA \& E. SOBRINO -2003-Plants invaders in Spain. (Check-list)
The Unwanted Citizens. http://www.medalienplants.org/checklist.pdf

GARCÍA MARTÍNEZ, X.M. -1985- Algunas aportaciones a la flora gallega. Anales Jard. Bot. Madrid 42(1): 191-196.

LAÍNZ, M. y E. LORIENTE -1983- Contribuciones al conocimiento de la flora montañesa, II. Anales Jard. Bot. Madrid 39(2): 405-416.

RYBNCEK, O. \& S. JÄGER -2001- Ambrosia (Ragweed) in Europe. Allergy Clin Immunol Int: $J$ World Allergy Org, 13(2): 60-66.

SANS ELORZA, M., E.D. DANA SÁNCHEZ y E. SOBRINO VESPERINAS -2004- Atlas de las plantas alóctonas invasoras en España. Ministerio de Medio Ambiente, Madrid.

Aceptado para su publicación en octubre de 2006

Dirección de los autores: Dpto. de Biología Vegetal. Universidad de Málaga. Apdo. 59.29080 Málaga (Spain). aerox@uma.es

\title{
122. DOS NOVEDADES COROLÓGICAS IMPORTANTES DE ORQUÍDEAS RARAS EN EL SURESTE IBÉRICO
}

\author{
José Antonio LÓPEZ ESPINOSA y Pedro SÁNCHEZ GÓMEZ
}

Two important chorological novelties of rare orchids in the south-east of the Iberian Peninsula

Palabras clave. Corología, sureste de España, orquídeas, Aceras anthropophorum, Epipactis microphylla.

Key words. Geographical distribution, south-east of Spain, orchids, Aceras anthropophorum, Epipactis microphylla.

Los estudios de seguimiento de la orquidoflora en la Región de Murcia, realizados en los últimos años, ponen de manifiesto que una proporción alta de los táxones de la familia indicados para la provincia son elementos raros de nuestra flora y con frecuencia de distribución muy puntual (López Espinosa \& Sánchez Gómez, 2006). 